\title{
Research on the Shrinkage of Model with Hole in PLA Material Based on the FDM 3D Printing
}

\author{
Quan Zhu', a, Yushan Liu, b, Yujun Cai ${ }^{1, c}$ and Meng $\mathrm{Wu}^{1, d}$ \\ ${ }^{1}$ Tianjin University of Technology \& Education., Tianjin, China, 300222 \\ a731073769@qq.com, byushan868@126.com, ${ }^{\circ}$ cyjal@126.com, ${ }^{d}$ wumeng5908@126.com
}

\section{Keywords: FDM; Shrinkage Hole; PLA; Orthogonal Experiments.}

Abstract. The accuracy of holes which are typical feature in various parts is an important requirement in 3D printing. In this paper, the influence of extrusion head temperature, layer thickness, line-width compensation, printing speed and other factors on the shrinkage of model with hole is researched in FDM process. And orthogonal experiments of printing the model with hole in PLA material is used to analysis the experimental results for getting the optimal combination of process parameters. Finally, the measure of reducing the shrinkage of model with hole is put forward.

\section{Introduction}

3D printing is a process of making a three dimensional solid object of virtually any shape from a digital model, which is achieved using an additive process on a professional device $[1,2]$. At present, 3D printing mainstream process is Fused Deposition Modeling (FDM), Stereo Lithography Apparatus (SLA), Selective Laser Sintering (SLS), etc. FDM is easy to operate, and the advantages of low cost are quickly popularized in the field of model design and production, teaching application [3].

The process of FDM technology involves layer-by-layer deposition of extruded material through a nozzle using feedstock filaments from a spool [4]. The forming principle is shown in Fig. 1.

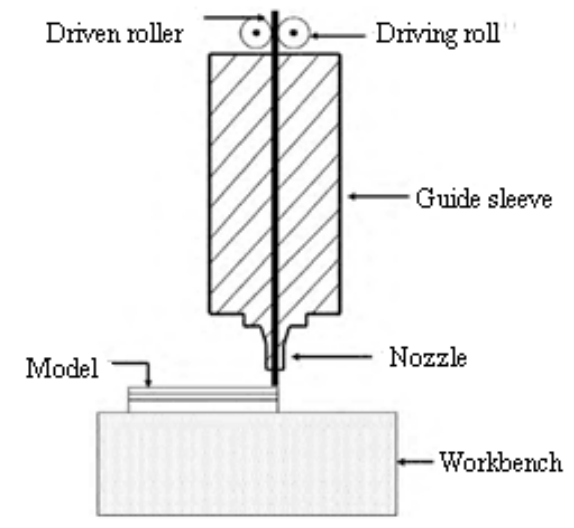

Fig. 1 The principle of fused deposition modeling

The phenomenon of the shrinkage of model that the size of printed model will be reduced compare to the theoretical size is found when printing the model in FDM process, which causes the printed model to be difficult to complete the assembly, but post-processes is needed to achieve assembly. The appearance of model is mainly square and round, the shrinkage hole is more complex compared to the shrinkage square according to experience and access to information, therefore, the shrinkage of model with hole is studied in this paper. PLA material is adopted for printing test, the optimum combination of process parameters are got which can effectively reduce the error of the shrinkage hole. Finally, the measures to reduce the error of size between the printed model with theoretical model is put forward.

\section{Factors of Affecting the Shrinkage Hole}

The factors of affecting the shrinkage hole are extrusion head temperature, hot bed temperature, layer thickness, extrusion speed, print speed in FDM process. 
(1) Extrusion head temperature. The effect of extrusion head device is to convert the solid wire into a molten state through high temperature. Heating temperature is set according to the different materials, such as PLA material is generally set to $190 \sim 230{ }^{\circ} \mathrm{C}$,ABS material is generally set to $180 \sim$ $250^{\circ} \mathrm{C}$. Extrusion head temperature is not conducive to printing when it is too high or too low, For example, the temperature once over $240{ }^{\circ} \mathrm{C}$, not only the mechanical properties of the model is decreased but the printing parts is stuck with yellow-brown carbide particles which is difficult to clear [5].

(2) Hot bed temperature. The shrinkage of model will be affected according to the temperature difference between hot bed with model. When its temperature is higher, the thermal stress of material is reduced that can avoid warping deformation, but the quality of surface is worse as wrinkling; the temperature is low, the thermal stress increases, resulting in warping deformation. Hot bed temperature is generally set in the range of $20 \sim 70{ }^{\circ} \mathrm{C}$.

(3) Layer thickness. This parameter is related to the nozzle diameter of the printer that generally is 0.1 to $0.4 \mathrm{~mm}$. If layer thickness value is large, effect of the step is obvious, poor print quality; or if layer thickness is small, the quality of printing model is high, but time-consuming. The printing model temperature is evenly distributed to avoid the phenomenon of shrinkage unevenness in the printing process when reducing the thickness [6].

(4) Extrusion speed. Extrusion speed refers to the speed of the wire from the nozzle extrusion, which is determined by the feeding speed and the extrusion pressure. If the speed of wire is too low, there is not enough silk to enter the nozzle; or if the speed of wire feeding is too fast, the extrusion speed of the extruded mechanism cannot keep up with the wire feeding speed, causing the fuse to pile up and the silk material is twisted and broken [7].

(5) Print speed. In the printing process, if the printing speed is too high, much faster than extrusion speed, the phenomenon of wire drawing will be occurred; or if the printing speed is too low, not only the processing time is increased, but also the quality of processed surface is affected.

\section{Experimental design and result analysis}

\section{Printing materials, manufacturing models and equipment}

The PLA material is selected for the printing materials, which is biodegradable. The ring structure is used for the manufacturing model in the experiment, as shown in Fig. 2. Three-arm parallel structure printer is used for the printing equipment, the nozzle diameter is $0.4 \mathrm{~mm}$, the wire diameter is $1.75 \mathrm{~mm}$.
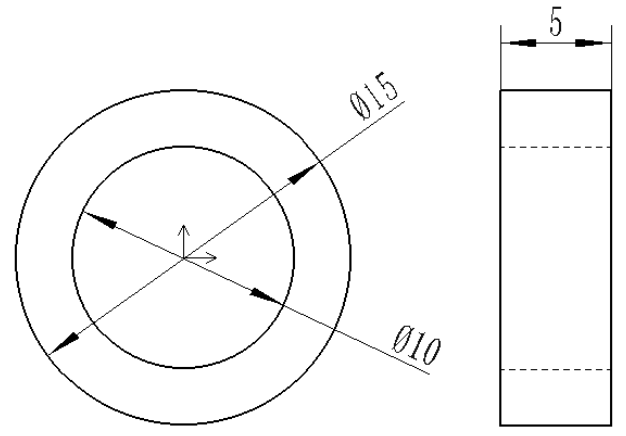

Fig. 2 Experimental model

\section{Orthogonal test}

Orthogonal test is the design method to study the multi-factor level. According to the orthogonality, the representative points are selected from all experiments to be tested, which have the characteristics of economy and efficiency [8].

There are many factors that affect the shrinkage of model with hole and the levels of different factors are not the same. Therefore, several factors such as extrusion head temperature, hot bed temperature, layer thickness and printing speed are selected as the influencing factors [9], three levels is selected for 
each factor. If all parameters are tested, it would theoretically require $4^{3}=64$ experiments, which is obviously undesirable. According to the level table of orthogonal test factor, nine experiments are carried out in this paper. The orthogonal test is described as following.

The table of test factors is shown in Table 1.

Table 1

The values of orthogonal test factors for model with hole

\begin{tabular}{cccc}
\hline \multicolumn{1}{|l}{ Impact factor } & level1 & level2 & level3 \\
\hline $\begin{array}{c}\text { Extrusion head } \\
\text { temperature ( A ) }{ }^{\circ} \mathrm{C}\end{array}$ & 190 & 200 & 210 \\
$\begin{array}{c}\text { Hot bed temperature } \\
\text { ( B ) }{ }^{\circ} \mathrm{C}\end{array}$ & 45 & 50 & 60 \\
$\begin{array}{c}\text { Layer thickness ( C ) } \\
\mathrm{mm}\end{array}$ & 0.1 & 0.2 & 0.3 \\
Print speed ( D ) mm/s & 40 & 45 & 50 \\
\hline
\end{tabular}

According to the orthogonal test table, the design of orthogonal test is obtained, and the printer was used to print 9 sets of the model with hole, each group printed 5 times, Hexagon coordinate measuring machine is used to measure, the average of 5 times as the final experimental data for each group, as shown in Table 2.

Table 2

The orthogonal test data of model with hole

\begin{tabular}{ccccccc}
\hline \multirow{2}{*}{ The no. } & \multicolumn{3}{c}{ Impact factor } & \multicolumn{3}{c}{ Experimental results } \\
\cline { 2 - 7 } & A & B & C & D & $\begin{array}{r}\text { Measured } \\
\text { value }(\mathrm{mm})\end{array}$ & $\begin{array}{c}\text { Size error } \\
(\mathrm{mm})\end{array}$ \\
\hline 1 & 190 & 45 & 0.1 & 40 & 9.78 & 0.22 \\
2 & 190 & 50 & 0.2 & 45 & 9.62 & 0.38 \\
3 & 190 & 60 & 0.3 & 50 & 9.50 & 0.50 \\
4 & 200 & 45 & 0.2 & 50 & 9.62 & 0.38 \\
5 & 200 & 50 & 0.3 & 40 & 9.52 & 0.48 \\
6 & 200 & 60 & 0.1 & 45 & 9.74 & 0.26 \\
7 & 210 & 45 & 0.3 & 45 & 9.60 & 0.40 \\
8 & 210 & 50 & 0.1 & 50 & 9.80 & 0.20 \\
9 & 210 & 60 & 0.2 & 40 & 9.66 & 0.34 \\
\hline
\end{tabular}

According to the data in Table 2, the mean and the difference of the dimensional error under different factor levels are calculated by the range analysis method, as shown in Table 3.

Table 3

The error mean of experimental data

\begin{tabular}{ccccc}
\hline \multirow{2}{*}{$\begin{array}{c}\text { Experimental } \\
\text { error }\end{array}$} & \multicolumn{4}{c}{ Impact factor } \\
\cline { 2 - 5 } & $\mathrm{A}$ & $\mathrm{B}$ & $\mathrm{C}$ & $\mathrm{D}$ \\
\hline Average1 & 0.36 & 0.32 & 0.22 & 0.35 \\
Average2 & 0.37 & 0.35 & 0.36 & 0.34 \\
& & & 0.46 & 0.36 \\
Average3 & 0.31 & 0.36 & 0.24 & 0.02 \\
poor & 0.06 & 0.04 &
\end{tabular}




\section{Analysis of experimental results}

The physical model printed is shown in Fig. 3. Green material is PLA.

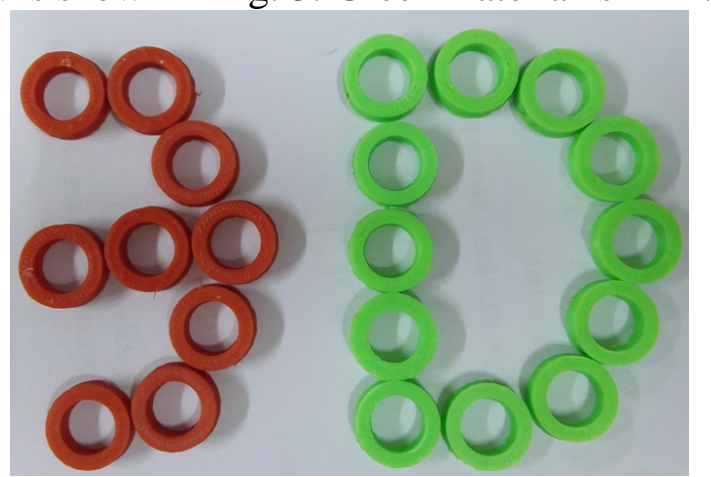

Fig. 3 Experimental model

According to the orthogonal experiment above, the optimal combination of process parameters can be obtained, which is $\mathrm{A}_{3} \mathrm{~B}_{1} \mathrm{C}_{1} \mathrm{D}_{2}$.

\section{Measures to reduce the shrinkage of model with hole}

The shrinkage of model with hole is related to extrusion head temperature, hot bed, layer thickness and printing speed, which could be reduced effectively according to the optimal combination of process parameters.

The wire has a certain width in printing process, so the actual fill contour will go beyond the theoretical profile while the nozzle moves in accordance with the theoretical contour trajectory [10], which is approximately half the diameter of the printed wire. For the final print parts, the outline dimensions will be larger than the actual size, the size of hole is smaller than the actual size. The principle of line-width compensation is shown in Fig. 4 and Fig. 5.

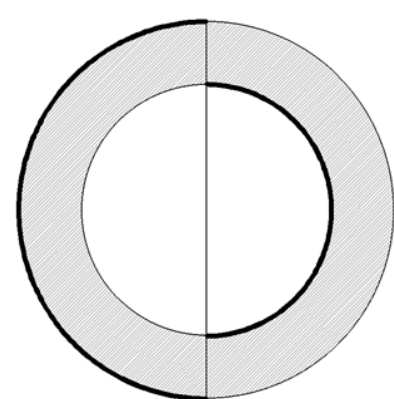

Fig. 4 Line width compensation is not used

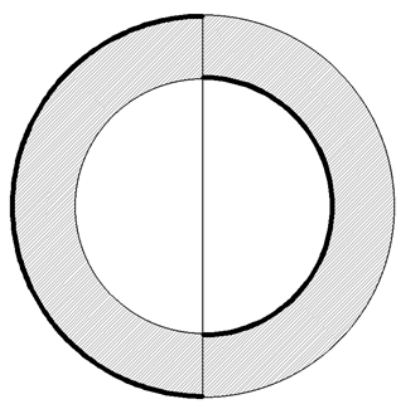

Fig. 5 Line width compensation has been used

The line-width compensation can be selected to reduce the shrinkage of model with hole according to the type of printed material in FDM process. For example, PLA material, once a sample size of $10 \mathrm{~mm}$ is selected, the line-width compensation value can be set to 0.36 , the printing parameters can be set according to the optimal combination of process parameters, finally the model size is set to $10.36 \mathrm{~mm}$, and the lasted measured value of printing model is $9.96 \mathrm{~mm}$. The final size of the sample subjected to the line-width compensation process is close to the theoretical size can be showed according to the above test.

\section{Conclusions}

The factors of affecting the shrinkage hole are extruded head temperature, hot bed temperature, layer thickness and printing speed when printing the model with hole. In this paper, the orthogonal test of 4 factors 3 levels is designed, and the optimal combination of process parameters of reducing the 
shrinkage error of model with hole is obtained which is $A_{3} B_{1} C_{1} D_{2}$ that extruded head temperature is $210{ }^{\circ} \mathrm{C}$, hot bed temperature is $45^{\circ} \mathrm{C}$, layer thickness is $0.1 \mathrm{~mm}$, printing speed is $45 \mathrm{~mm} / \mathrm{s}$.

Finally, the concrete measure to reduce the shrinkage of model with hole is put forward, which is practical significance to solve the problem of model with hole assembly in FDM process.

\section{Acknowledgements}

This work was financially supported by the Key Technology Research and Development Program of Tianjin(13ZCZDGX01108, 15PTYJGX00050).

\section{References}

[1] LU Bing-heng. Research progress of advanced manufacturing technology in Xi'an Jiaotong University[J]. Engineering Science,2013,(1):4-8.

[2] M Wonjin Jo, Jang Hee L, et al. Introduction of 3D Printing Technology in the Classroom for Visually Impaired Students[J]. Journal of Visual Impairment \& Blindness,2016,110(2):115-121.

[3] SONG ke, YANG Bang-cheng. Example Application of 3D Printing Technology [J] .New Technology \& Technology, 2015, (12): 90-93.

[4] YAN Ming. Structure Design and Optimization of FDM Rapid Prototyping Machine [D]. Wuhan: Hua Zhong University of Science and Technology, 2009.

[5] LIU Xin-hua, LI Sheng-peng, LIU Zhou, et al. An investigation on distortion of PLA thin-plate part in the FDM process[J].International Journal of Advanced Manufacturing Technology,2015,79(5):1117-1126.

[6] LU Jian-hui. Rapid forming precision and process research of molten deposition [D]. Dalian: mechanical manufacturing and automation college, dalian university of technology, 2009.

[7] Jelena Zarko, Gojko Vladic, Magdolna Pal, et al. Influence of printing speed on production of embossing tools using FDM 3D printing technology[J].Journal of Graphic Engineering \& Design,2017,8(1):19-27.

[8] Omar Ahmed Mohamed, Syed Hasan Masood, Jahar Lal Bhowmik. Mathematical modeling and FDM process parameters optimization using responsesurface methodology based on Q-optimal design[J]. Applied Mathematical Modelling,2016,40:10052-10073.

[9] Gurrala Pavan Kumar, Regalla Srinivasa Prakash. Multi-objective optimisation of strength and volumetric shrinkage of FDM parts[J]. Virtual \& Physical Prototyping,2014,9(2):127-138.

[10] Sood Anoop K, Ohdar Raj K, Mahapatra Siba S. Experimental investigation and empirical modelling of FDM process for compressive strength improvement[J]. Journal of Advanced Research,2012,3(1):81-90. 